\title{
Effect of alcohols on gastric and small intestinal apical membrane integrity and fluidity
}

\author{
HEATHER J BALLARD, J M WILKES, AND B H HIRST \\ From the Department of Physiological Sciences, University of Newcastle upon Tyne Medical School, Newcastle \\ upon Type
}

SUMMARY Duodenal and jejunal brush border membrane vesicle integrity was studied after in vitro treatment of rabbit tissue with ethyl, benzyl or octyl alcohol. The effects of the alcohols on gastric parietal cell apical and microsomal membrane vesicle integrity was also studied. Membrane vesicle integrity was determined from the enclosed volume of the vesicle preparations, measured as $\left[{ }^{14} \mathrm{C}\right]$ glucose space at equilibrium. Exposure of vesicles to the three alcohols caused concentration dependent decreases in enclosed volume. The rank order of potency of the alcohols was octyl> benzyl>ethyl. Concentrations $\geqslant 10 \mathrm{mM}$ benzyl alcohol significantly reduced the enclosed volume of duodenal or jejunal vesicles; jejunal vesicles were disrupted by $625 \mathrm{mM}$ ethanol, whereas $2 \mathrm{M}$ ethanol was required to disrupt the duodenal vesicles. Gastric apical membrane integrity was reduced with $0 \cdot 25 \mathrm{M}$ ethanol, the vesicles being approximately an order of magnitude more sensitive to ethanol than gross estimates of gastric mucosal damage, but $1 \mathrm{M}$ ethanol was required to significantly damage gastric microsomes. All concentrations of benzyl or octyl alcohol tested $(\geqslant 5 \mathrm{mM})$ reduced the enclosed volume of both gastric apical membrane vesicles and gastric microsomes. As determined by shrink-swell techniques, benzyl alcohol permeated duodenal vesicles at a faster rate than $\mathrm{NH}_{4} \mathrm{Cl}$ (apparent rate constant of $9.89(0 \cdot 71) \times 10^{-3} \mathrm{~s}^{-1}$ compared with 4.48 $\left.(0 \cdot 23) \times 10^{-3} \mathrm{~s}^{-1}\right)$. Therefore, reductions in enclosed volume in response to alcohol treatment could not be explained by alcohol induced osmotic shrinkage. The enclosed volume of the vesicles after alcohol treatment was negatively correlated with membrane fluidity suggesting a common causal effect, the increased fluidity increasing membrane fragility. Duodenal vesicles were more resistant to disruption by the alcohols compared with gastric and jejunal vesicles.

Chronic alcoholics frequently suffer from malnutrition. It has been suggested that continued exposure to ethanol may impair intestinal absorption, and contribute to this malnutrition. Acute administration of ethanol has been shown to inhibit the absorption of glucose and amino acids, both in vivo ${ }^{12}$ and in vitro. ${ }^{34}$ Several possible mechanisms may contribute to this inhibition: (i) the permeability of the apical surface of the cell to sodium may be increased, allowing the intracellular concentration to rise and dissipate the driving force for transport; (ii) ethanol may directly inhibit the transport molecules for these solutes; (iii)

Address for correspondence: Dr Barry H Hirst, Dept of Physiological Sciences, The Medical School, Framlington Place, Newcastle upon Tyne NE2 4HH. Received for publication 8 June 1988.
$\mathrm{Na}^{+} / \mathrm{K}^{+}$-ATPase may be inhibited; (iv) ethanol may physically damage the absorptive cells, producing changes in the membrane fluidity or chemical composition. Experiments in rat or hamster jejunal brush border membrane (BBM) vesicles indicated that there were no direct effects of ethanol on the $\mathrm{Na}^{+}-$ driven transport of glucose or amino acids, but that ethanol exerted a dual effect on transport by increasing membrane conductance for $\mathrm{Na}^{+}$and decreasing intravesicular volume. ${ }^{56}$ Both these reported effects could be mediated through a change in membrane fluidity.

The present experiments were undertaken to determine the effects of ethanol induced changes in membrane fluidity on membrane integrity, using duodenal and jejunal BBM vesicles. To help in 
distinguishing any specific effects of ethanol from those mediated through fluidity changes, the effects of benzyl and octyl alcohols on membrane fluidity and integrity were also investigated. In addition, the effects of the alcohols on membrane integrity of apical membrane vesicles from the gastric parietal cell (so called stimulation associated vesicles), and gastric microsomes were investigated. Ethanol, and other alcohols, are damaging to the gastric mucosa, and break the gastric mucosal barrier..$^{7-4}$

The enclosed volume of the vesicle preparation, determined as $\left[{ }^{14} \mathrm{C}\right]$ glucose space at equilibrium, was used as a measure of the membrane integrity: disruption of some of the vesicles would reduce the total enclosed volume of the preparation. Exposure to increasing concentrations of the alcohols might, however, instead, cause osmotic shrinkage of the vesicles. This possibility was investigated by determining the rate at which benzyl alcohol was able to permeate the duodenal vesicles.

\section{Methods}

ANIMALS

Young New Zealand white rabbits $(\sim 3 \mathrm{~kg})$ of either sex were anaesthetised by iv injection of sodium pentobarbital. Brush border membrane vesicles were prepared from the duodena (first $40 \mathrm{~cm}$ of small intestine) or the jejuna (middle $40 \mathrm{~cm}$ of small intestine) of the rabbits by a slight modification of the method of Kessler et al: : $^{11}$ briefly, small intestinal mucosal scrapings were suspended in a medium containing $50 \mathrm{mM}$ mannitol, $2 \mathrm{mM}$ Tris $/ \mathrm{HCl}(\mathrm{pH}$ $7 \cdot 1$ ), and homogenised for two minutes at a setting of 200 in a Servall Omni Mixer. Magnesium chloride (1 M) was added to a final concentration of $10 \mathrm{mM}$, and the mixture was stirred on ice for 15-20 minutes, then centrifuged for 20 minutes at $3000 \mathrm{~g}$. The pellet was discarded and the supernatant centrifuged for 40 minutes at $27000 \mathrm{~g}$. The new pellet, which contained the $\mathrm{BBM}$ vesicles, was resuspended in $300 \mathrm{mM}$ mannitol, $10 \mathrm{mM}$ Tris/ $\mathrm{HCl}(\mathrm{pH} 7 \cdot 1)$, and centrifuged for 20 minutes at $5000 \mathrm{~g}$. The pellet was discarded, and the purified BBM vesicles were obtained by centrifuging the supernatant at $27000 \mathrm{~g}$ for 40 minutes. The vesicles in the pellet were resuspended in $300 \mathrm{mM}$ mannitol, $10 \mathrm{mM}$ Tris/ $\mathrm{HCl}(\mathrm{pH} 7 \cdot 1)$, at a protein concentration of $10 \mathrm{mg} / \mathrm{ml}$.

Gastric vesicles were prepared from the stomachs of the rabbits as previously described by Hirst and Forte: ${ }^{11}$ either parietal cell apical membrane vesicles were prepared from stomachs stimulated to secrete acid (animals fed and pretreated with histamine) or gastric microsomes were prepared from unstimulated stomachs (animals fasted overnight and pretreated with cimetidine). In either case, fundic mucosal scrapings were placed in a medium containing 125 $\mathrm{mM}$ mannitol, $40 \mathrm{mM}$ sucrose, $1 \mathrm{mM}$ EDTA, $5 \mathrm{mM}$ Pipes/Tris ( $\mathrm{pH} 7 \cdot 1$ ), minced with scissors, and homogenised by 16-18 passes of a PotterElvehjem homogeniser at $200 \mathrm{rev} / \mathrm{min}$. The homogenate was centrifuged at $800 \mathrm{~g}$ for 10 minutes and the pellet discarded. To obtain parietal cell apical membrane vesicles, the supernatant was centrifuged at $7000 \mathrm{~g}$ for 12 minutes; the pellet from this spin was resuspended in $300 \mathrm{mM}$ sucrose, $5 \mathrm{mM}$ Tris $/ \mathrm{HCl}$ ( $\mathrm{pH} 7 \cdot 4)$, and fractionated on a discontinuous Ficoll gradient at $90000 \mathrm{~g}$ for 16 hours. The parietal cell apical membrane vesicles were collected from the interface between $10 \%$ and $16 \%$ Ficoll, diluted with 25-50 vol of the sucrose-Tris solution, and harvested by centrifugation at $20000 \mathrm{~g}$ for one hour. These vesicles were resuspended in the sucrose-Tris solution and used at a protein concentration of 1-2 $\mathrm{mg} / \mathrm{ml}$. To obtain gastric microsomes, the supernatant from the first $(800 \mathrm{~g})$ centrifugation was recentrifuged at $2000 \mathrm{~g}$ for 10 minutes, then at 12000 $g$ for 12 minutes, the pellet being discarded in both cases. The $12000 \mathrm{~g}$ supernatant was centrifuged at $135000 \mathrm{~g}$ for 60 minutes, and the pellet resuspended in the sucrose-Tris solution and fractionated on a discontinuous Ficoll gradient for 16 hours at $90000 \mathrm{~g}$. The material at the interface between 0 and $5 \%$ Ficoll was collected, diluted and harvested by centrifuga-

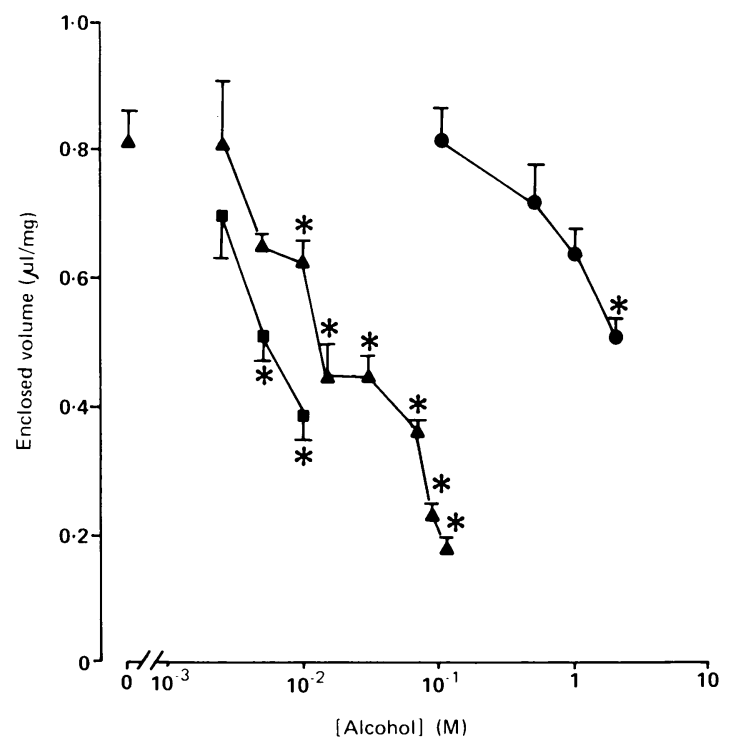

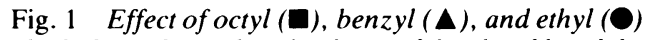
alcohols on the enclosed volume of duodenal brush border membrane vesicles. Vesicles were equilibrated for 45 minutes in the presence of $/{ }^{14} \mathrm{C} /$ glucose, and various concentrations of one of the alcohols. Values are the mean (SE) of 4-26 tests; *, $p<0.05$ compared with control. 
tion at $135000 \mathrm{~g}$ for one hour. Gastric microsomes were used at a protein concentration of $2-4 \mathrm{mg} / \mathrm{ml}$.

The total enclosed volume of the vesicles in the presence of various concentrations of ethyl, benzyl, or octyl alcohol was found by the retention of $\left[{ }^{14} \mathrm{C}\right]$ glucose at equilibrium (45 minutes for small intestinal vesicles or 75 minutes for gastric vesicles): aliquots of the vesicles were diluted into an equal volume of a medium containing $150 \mathrm{mM} \mathrm{NaCl}, 200$ $\mathrm{mM}$ Hepes ( $\mathrm{pH} \mathrm{7.4),0.2} \mathrm{mM} \mathrm{glucose} \mathrm{and} 1.2$ $\mathrm{MBq} / \mathrm{ml} \mathrm{D}-\left[\mathrm{U}-{ }^{14} \mathrm{C}\right]$ glucose, and the test alcohol. After incubation at room temperature, the vesicles were harvested onto $0.45 \mu \mathrm{m}$ nitrocellulose filters (Biotrace NT, Gelman Sciences Inc.) and washed with two $5 \mathrm{ml}$ aliquots of $145 \mathrm{mM} \mathrm{NaCl}, 0.5 \mathrm{mM}$ phloridzin. The filters were allowed to dry and $5 \mathrm{ml}$ Optiphase Safe (LKB) added before liquid scintillation counting. Enclosed volume was expressed as $\mu \mathrm{l} /$ $\mathrm{mg}$ vesicular protein.

The rates at which benzyl alcohol, $\mathrm{NH}_{4} \mathrm{Cl}$ and $\mathrm{NaCl}$ permeated the duodenal vesicles were found by a shrink-swell technique ${ }^{12}$ in which the light scattered by the vesicles was taken as a measure of their size: thus the rate of reswell after osmotic shrinkage because of the addition of solute could be followed. The vesicles were diluted to a protein concentration of $0.25 \mathrm{mg} / \mathrm{ml}$ in $300 \mathrm{mM}$ mannitol, $10 \mathrm{mM}$ Tris/ $\mathrm{HCl}(\mathrm{pH} 7 \cdot 1) ; 1.5 \mathrm{ml}$ of this suspension were placed in a cuvette and illuminated with $450 \mathrm{~nm}$ light in a fluorimeter (Perkin Elmer LS5). The $450 \mathrm{~nm}$ light

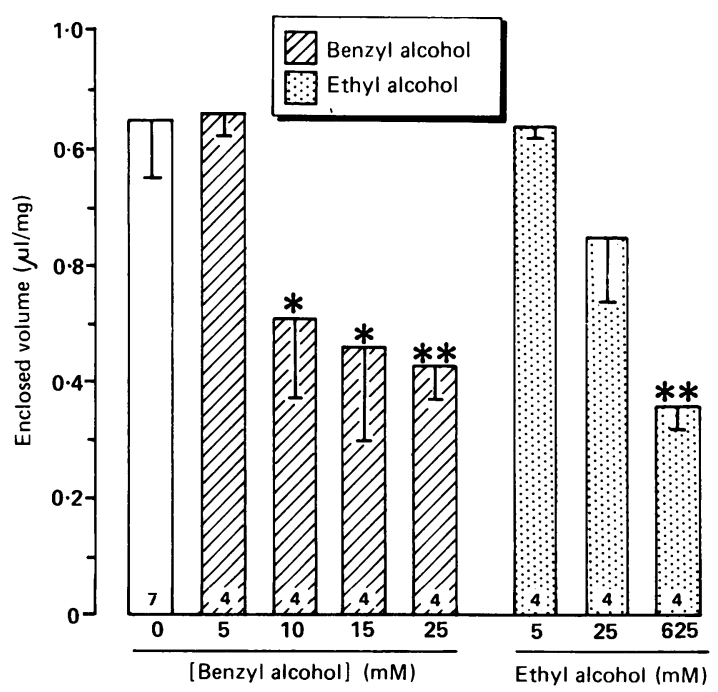

Fig. 2 Effect of benzyl and ethyl alcohols on the enclosed volume of jejunal brush border membrane vesicles. Vesicles were equilibrated for 45 minutes in the presence $\left[{ }^{14} \mathrm{C} / g\right.$ lucose, and various concentrations of benzyl or ethyl alcohol. Values are the mean (SE) of the number of tests shown on each bar; ${ }^{*}, p<0.05 ;{ }^{* *}, p<0.005$ compared with control. scattered by the vesicle suspension was monitored at $90^{\circ}$, whilst $1.5 \mathrm{ml}$ of a solution containing 250 $\mathrm{mOsm} / 1$ of the test solute in $300 \mathrm{mM}$ mannitol 10 $\mathrm{mM}$ Tris, $\mathrm{pH} 7 \cdot 1$ were added and mixed. The change in light scattering was then monitored for a further 25 minutes.

The fluidity of the lipid regions of the vesicle membranes in the presence or absence of each of the alcohols was determined at $20^{\circ} \mathrm{C}$ from the fluorescence anisotropy ( $r$ ) of the probe 1,6-diphenyl1,3,5-hexatriene, as described by Shinitzky and Barenholz, ${ }^{13}$ with conditions as described by Wilkes et al. ${ }^{14}$ Fluidity was expressed in terms of the parameter $\left(r_{0} / r\right)-1$, where $r_{o}$ is the limiting fluorescence anisotropy. Protein was determined by the Bradford method, ${ }^{15}$ using $\gamma$-globulin as a standard.

Significance of difference between mean values was investigated by analysis of variance followed by Student's $t$ test. Significance was set at $\mathrm{p}<0 \cdot 05$. Correlation lines were calculated by the method of least squares.

\section{Results}

EFFECT OF ALCOHOLS ON ENCLOSED VOLUME OF SMALLINTESTINAL BBM VESICLES

Duodenal BBM vesicles were isolated with a total enclosed volume of $0.81(0.05) \mu \mathrm{l} / \mathrm{mg}$ (mean (SE), $\mathrm{n}=26$ ). Exposure of the vesicles to a range of benzyl alcohol concentrations for 45 minutes at room temperature resulted in dose dependent decreases in their total enclosed volume, as assessed from the retention of $\left[{ }^{14} \mathrm{C}\right]$ glucose at equilibrium (Fig. 1). Concentrations of benzyl alcohol greater than or equal to $10 \mathrm{mM}$ were required to significantly reduce the enclosed volume.

Concentrations of ethyl alcohol up to and including $1 \mathrm{M}$ did not alter the total enclosed volume of the duodenal BBM vesicles (Fig. 1). Equilibration with $2 \mathrm{M}$ ethanol, however, reduced the enclosed volume of the duodenal BBM vesicles by about $30 \%$. All of the concentrations of octyl alcohol tested $(2 \cdot 5-$ $10 \mathrm{mM}$; Fig. 1) produced significant reductions in vesicular volume. The rank order of potency of the alcohols in reducing vesicle volume was thus octyl> benzyl>ethyl: similar reductions in enclosed volume were produced by $2.5 \mathrm{mM}$ octyl, $30 \mathrm{mM}$ benzyl and $2 \mathrm{M}$ ethyl alcohol.

The total enclosed volume of the jejunal BBM vesicles was $0.85(0.08) \mu \mathrm{l} / \mathrm{mg}(\mathrm{n}=7$; not significantly different from that of duodenal BBM vesicles). Exposure to ethyl or benzyl alcohol produced dosedependent decreases in total enclosed volume of jejunal BBM vesicles (Fig. 2): concentrations greater than or equal to $10 \mathrm{mM}$ benzyl alcohol or $625 \mathrm{mM}$ ethyl alcohol significantly reduced vesicular volume. 

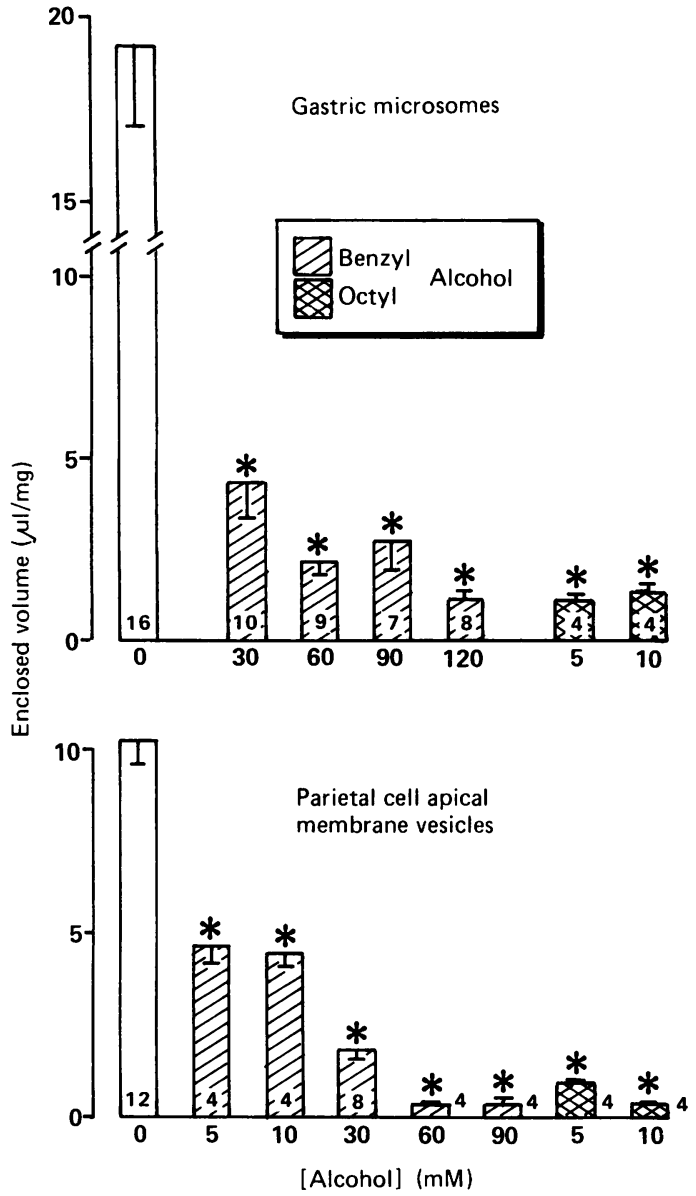

Fig. 3 Effect of benzyl and octyl alcohols on the enclosed volume of gastric microsomes and parietal cell apical membrane vesicles. Vesicles were equilibrated for 75 minutes in the presence of $\left[{ }^{14} \mathrm{C}\right] \mathrm{glucose}$, and various concentrations of benzyl or octyl alcohol. Values are the mean (SE) of the number of tests shown on each bar; ${ }^{*}, p<0.001$ compared with control.

EFFECT OF ALCOHOLS ON ENCLOSED VOLUME OF GASTRIC MEMBRANE VESICLES

Parietal cell apical membrane vesicles were isolated with a total enclosed volume of $10.22(0.64) \mu \mathrm{l} / \mathrm{mg}$ $(n=12)$. All of the concentrations of benzyl $(\geqslant 5$ $\mathrm{mM})$ or octyl alcohol $(\geqslant 5 \mathrm{mM}$; Fig. 3) tested produced significant reductions in total enclosed volume, whilst ethyl alcohol produced dosedependent decreases in enclosed volume (Fig. 4). The threshold concentration for ethyl alcohol reduction of enclosed volume was $0.25 \mathrm{M}$ (Fig. 4).

Gastric microsomes had a total enclosed volume of $19 \cdot 24(2 \cdot 18) \mu \mathrm{l} / \mathrm{mg}(\mathrm{n}=16)$. Significant reductions in total enclosed volume were produced by all the
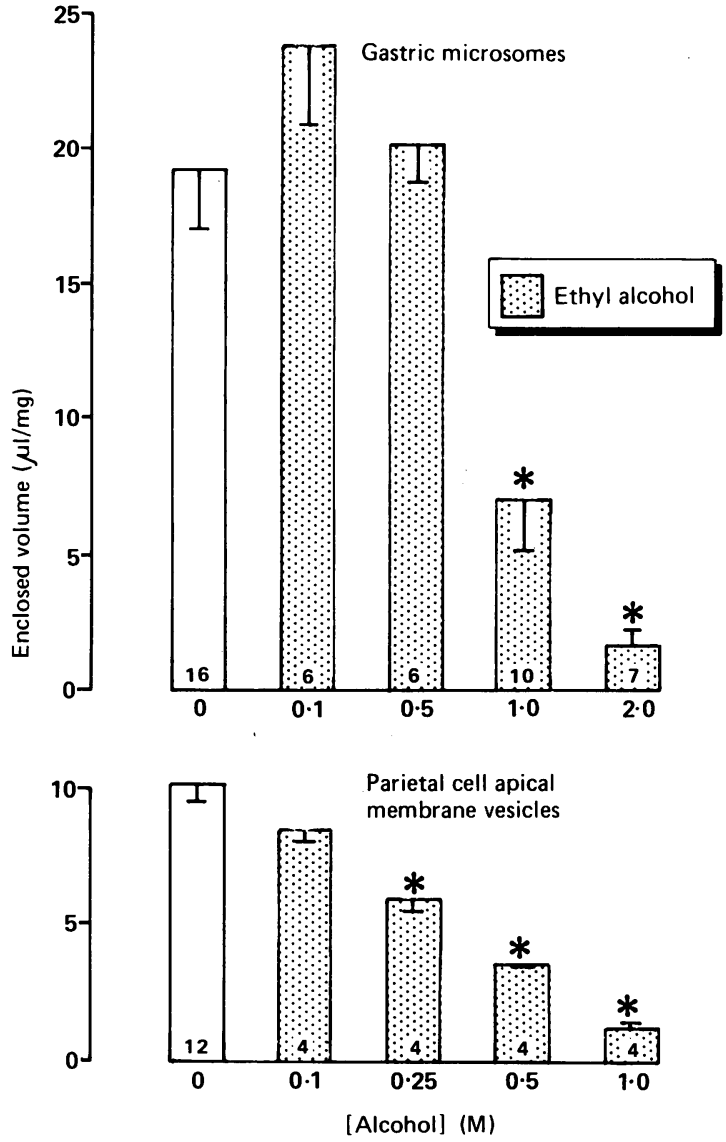

Fig. 4 Effect of ethyl alcohol on the enclosed volume of gastric microsomes and parietal cell apical membrane vesicles. Vesicles were equilibrated for 75 minutes in the presence of $\left[{ }^{14} \mathrm{C}\right]$ glucose, and various concentrations of ethyl alcohol. Values are the mean (SE) of the number of tests shown on each bar; ${ }^{*}, p<0.005$ compared with control.

concentrations of benzyl alcohol $(\geqslant 30 \mathrm{mM})$ or octyl alcohol ( $\geqslant 5 \mathrm{mM}$; Fig. 3$)$ tested, and by concentrations of ethyl alcohol $\geqslant 1 \mathrm{M}$ (Fig. 4).

CORRELATION BETWEEN MEMBRANE FLUIDITY AND TOTAL ENCLOSED VOLUME OF VESICLES The fluidity of the hydrophobic regions of the duodenal BBM $((\mathrm{r} / \mathrm{r})-1)$ under control conditions was $0.317(0 \cdot 024)(n=7)$. The jejunal BBM had a fluidity of $0.329(0.025) \quad(n=4)$, which was not significantly different from that of duodenal BBM. The fluidities of the gastric microsomal membrane and the parietal cell apical membrane were 0.462 $(0.023)(n=3)$ and $0.589(0.017)(n=4)$, respectively. The three alcohols raised membrane fluidity in a dose-dependent manner: the rank order of potency 


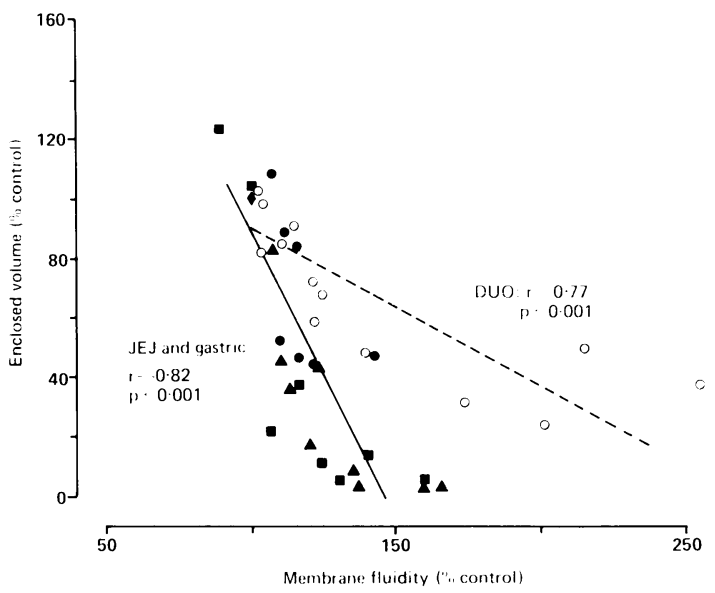

Fig. 5 Relationship between enclosed volume and membrane fluidity for duodenal brush border vesicles $(o$, dashed line) and for jejunal brush border vesicles (O), parietal cell apical membrane vesicles $(\boldsymbol{\Delta})$, and gastric microsomes $(\mathbf{\square})$, which all fell on the same correlation line (solid line). Enclosed volume and membrane fluidity are both normalised to the value for untreated vesicles $(\lambda)$. The two variables were altered by varying the concentrations of octyl, benzyl, and ethyl alcohols (Figs. I-4). was octyl $>$ benzyl $>$ ethyl. These data are reported elsewhere. ${ }^{1+16}$ The enclosed volume of the duodenal vesicle preparation following alcohol treatment was negatively correlated with the fluidity of the membrane (Fig. 5). Similarly, the enclosed volumes of the jejunal, gastric microsomal and parietal cell apical membrane vesicle preparations after alcohol treatment were also correlated with their membrane fluidities: the normalised volume versus fluidity data for the latter three preparations fell on a single correlation line which was steeper than the line relating volume to fluidity for the duodenal vesicles (Fig. 5).

RATE OF PERMEATION OF BENZYL ALCOHOL INTO DUODENAL BBM VESICLES

The vesicles shrank immediately upon the addition of benzyl alcohol, which resulted in an increase in the light scattering (Fig. 6). The subsequent decrease in light scattering as the vesicles reswelled consisted of an initial exponential component superimposed on a linear phase. The exponential phase of the change in light scattering $\left(\Delta F_{t}\right)$ with time $(t)$ (see inset Fig. 6) was linearised graphically by plotting $\ln \Delta \mathrm{F}_{\mathrm{t}}$

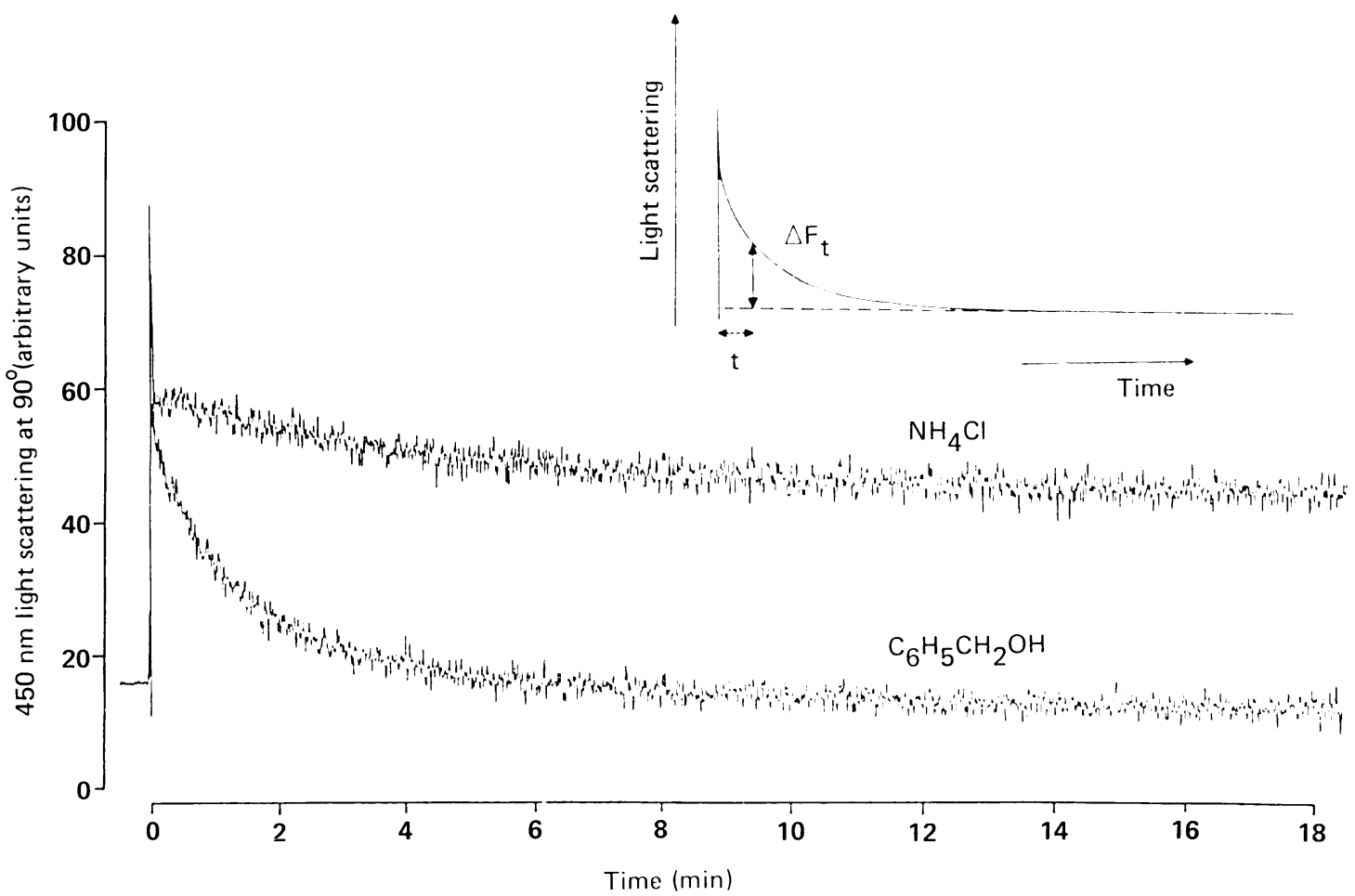

Fig. 6 Change in light scattered by duodenal brush border membrane vesicle suspension (0.25 $\mathrm{mg}$ protein/ml) with time after exposure to $250 \mathrm{mOsm} / \mathrm{l}$ benzyl alcohol or $\mathrm{NH}_{4} \mathrm{Cl}$. Inset: Method for measuring values of exponential phase of light scattering $\left(\Delta F_{t}\right)$ from experimental traces. 


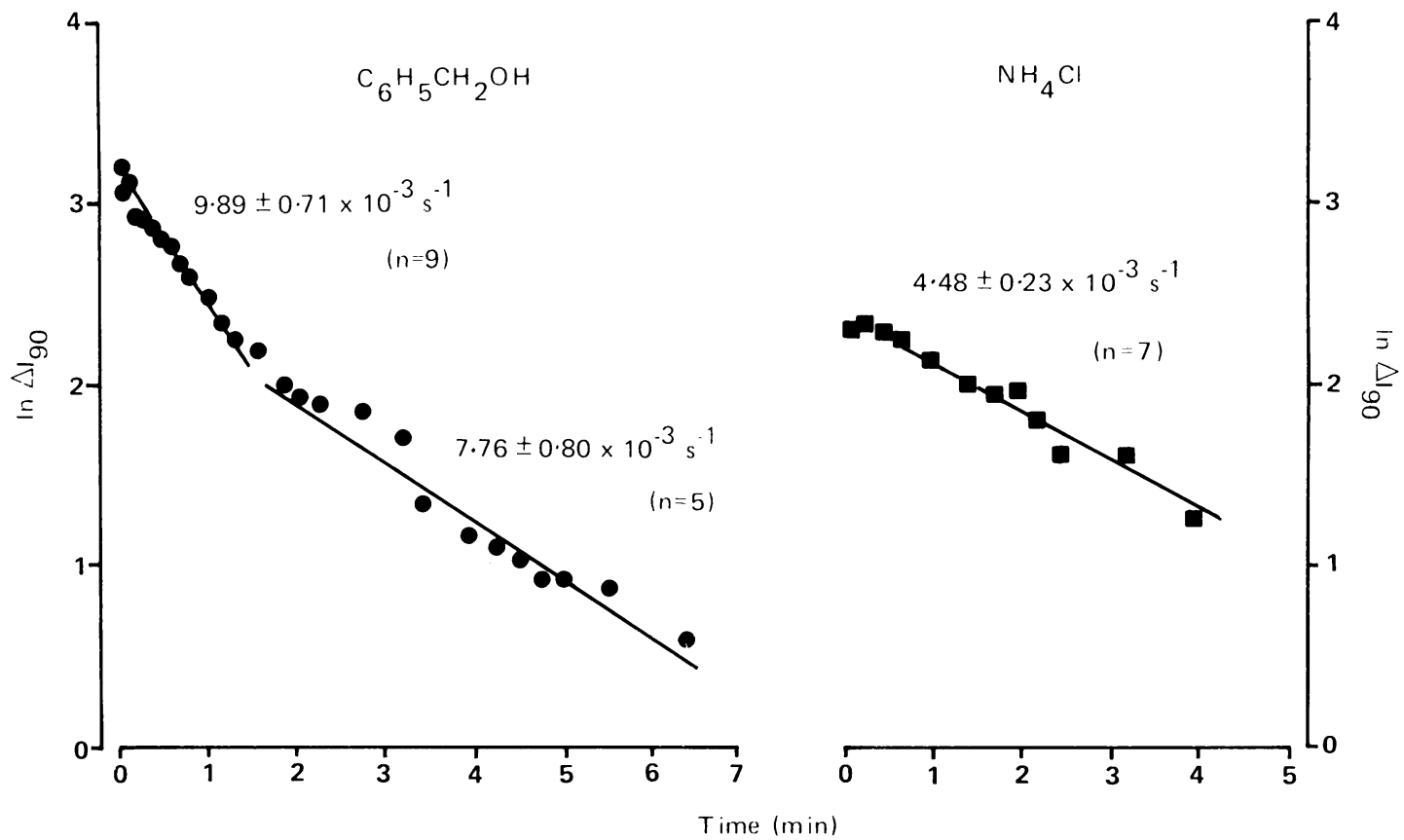

Fig. 7 Change in exponential component of light scattered at $90^{\circ} \mathrm{C}\left(\Delta I_{\aleph_{0}}\right.$ or $\left.\Delta F_{1}\right)$ with time. Rate constants for the entry of the solutes were calculated as the slopes of the lines relating $\ln \Delta \mathrm{I}_{\mathrm{O}_{1}}$ to time.

(comparable to $\ln \Delta \mathrm{I}_{\varphi_{0}}$ ) against time (Fig. 7). The first order apparant rate constant for the entry of the solute was calculated as the slope of the line relating $\ln \Delta \mathrm{F}_{\mathrm{t}}$ against time. Benzyl alcohol permeated the vesicles with two first order apparant rate constants of $9.89(0.71) \times 10^{-3} \mathrm{~s}^{-1}(\mathrm{n}=9)$ and $7.76(0.80) \times 10^{-3} \mathrm{~s}^{-1}$ $(\mathrm{n}=5$; Fig. 7). As a comparison, the rates of entry of the highly permeant species, $\mathrm{NH}_{4} \mathrm{Cl}$, and the relatively impermeant species, $\mathrm{NaCl}$, were also determined under similar conditions: $\mathrm{NH}_{4} \mathrm{Cl}$ permeated the vesicles with a single first order apparant rate constant of $4.48(0.23) \times 10^{-3} \mathrm{~s}^{-1}(\mathrm{n}=7$; Fig. 6 and 7). Over the timecourse of the experiment $(25 \mathrm{~min})$, $\mathrm{NaCl}$ did not permeate the vesicles. Thus the degree of light scattering increased upon $\mathrm{NaCl}$ addition, and remained constant at the new higher level.

\section{Discussion}

These data show that the three alcohols tested were capable of reducing enclosed volume in all of the types of vesicle examined. Osmotically induced volume changes still remaining after 45-75 minutes of incubation can be excluded, as benzyl alcohol was found to permeate the vesicles even more rapidly than a recognised permeant species, $\mathrm{NH}_{4} \mathrm{Cl}$, and reswelling of vesicles after shrinkage with benzyl alcohol was complete by about 15 minutes (Figs 6 and
7). In addition, high concentrations of ethanol, with a high osmotic potential, were relatively ineffective in reducing enclosed volume, whilst large reductions were seen with concentrations of benzyl and octyl alcohols of low osmotic potential. Thus, the volume changes observed in the present experiments represent the disruption of a proportion of the vesicles, and can therefore be used as a measure of membrane integrity.

A reduction in the enclosed volume of the vesicles, as seen in the present experiments, would be expected to reduce the equilibrium uptake of solutes such as glucose and amino acids. Previously, ethanol has been shown to inhibit sodium dependent uptake of glucose and alanine into jejunal BBM vesicles, but not to alter the equilibrium uptake of the solutes. " These authors, however, used concentrations of ethanol $(300-500 \mathrm{mM})$, which were below the threshold for volume reduction in the present experiments (Figs 1 and 2). They found no direct effects of ethanol on the transport processes, but the membrane conductance for $\mathrm{Na}$ ' was increased by ethanol. This is supported by previous observations from our own laboratory, that fluidising duodenal BBM with benzyl alcohol led to an increase in the passive permeability of the membrane to $\mathrm{NaCl}^{14}$ Similarly, the proton permeability of duodenal BBM and gastric membranes was increased by fluidising the 
membranes with low concentrations of octyl, benzyl, and ethyl alcohols. ${ }^{8146}$ Concentrations of ethanol greater than or equal to $1.6 \mathrm{M}$ were required to increase DNA liberation into intestinal perfusates. ${ }^{2}$ Green $e t a^{2}$ also reported that the in vivo absorption of amino acids from the perfused small intestine of the rat was not reduced until the mucosal ethanol concentration reached $2 \mathrm{M}$, in contrast with earlier in vitro findings that as little as $86 \mathrm{mM}$ ethanol will inhibit amino acid uptake. ${ }^{1}$ We suggest, therefore, that the effects of ethanol are two-fold: low concentrations raise the membrane conductance for $\mathrm{Na}^{+}$, dissipating the driving force for transport, while higher concentrations disrupt the membrane itself.

In the intact stomach, the threshold concentration of ethanol required to induce gross damage to the mucosa, including increased transmucosal flux of $\mathrm{H}^{+}$ and $\mathrm{Na}^{+}$, and reduced transmucosal potential difference, is around $2 \mathrm{M}^{{ }^{7-9}}$ Lower concentrations of ethanol, however $(0.5 \mathrm{M})$ increase membrane fluidity in intact gastric cells, ${ }^{17}$ and gastric microsomes and apical plasma membranes ${ }^{1418}$ associated with increased proton permeability. ${ }^{9}$ Thus, the stomach and gastric membranes behave in a comparable bi-phasic manner to the intestinal BBM in response to ethanol insult; increased membrane permeability and membrane disruption. Relatively low concentrations of ethanol $(0.25 \mathrm{M})$ reduced gastric parietal cell apical membrane vesicle integrity (Fig. 4), and thus these isolated membrane vesicles are a sensitive model for gastric mucosal damaging effects of barrier breaking agents.

Ethanol concentrations in the human gut reach 0.1-2 $M$ after moderate alcohol ingestion; ${ }^{919}$ undiluted spirits contain $\sim 8 \mathrm{M}$ ethanol. As expected, there is a gradation in the luminal concentrations of ethanol achieved after oral ingestion. For example, after oral administration of $0.8 \mathrm{~g} / \mathrm{kg}$ of a $25 \%$ ethanol solution $(5.4 \mathrm{M})$, peak gastric ethanol concentrations in man reached $1 \cdot 5-1 \cdot 7 \mathrm{M}$. In comparison, peak ethanol concentrations in the duodenum and proximal jejunum were $0 \cdot 2-1 \cdot 1 \mathrm{M}$, and in the midjejunum $80 \mathrm{mM}$. The maximum ethanol concentration observed in the ileum in the same experiments, $40 \mathrm{mM}$, was similar to that in the serum. ${ }^{19}$ Alcohol in the gut, and in particular in the stomach, can therefore be expected to reach concentrations high enough to induce cellular disruption and impair absorption of nutrients, if alcohol is consumed in excess.

The alcohol induced distruption of the vesicle membranes appears to result from their fluidising effect, rather than a specific chemical effect, as there was a single correlation line relating enclosed volume to membrane fluidity following treatment. We can postulate therefore, that the main effect of the raised fluidity is to increase membrane 'fragility', making the vesicles more susceptible to damage. The rank order for the potency of the alcohols to reduce the enclosed volumes of the membrane vesicles, octyl $>$ benzyl>ethyl (Figs 1-4), is a reflection of their oilwater partition coefficients. The same rank order is observed for increases in proton permeability in vitro ${ }^{16}$ and in vivo. ${ }^{8}$ The common linking factor may be the membrane fluidizing activity of the alcohols.

No differences were found for the increase in membrane fluidity required for membrane disruption between microsomal and the apical membranes from the stomach. The jejunal vesicles showed a similar degree of susceptibility to damage as the gastric vesicles, falling on a single correlation line with them, whereas the duodenal vesicles were much more resistant to disruption by the alcohols (Fig. 5). It is unclear from these experiments what confers this unique resistance to disruption by alcohols on the duodenal brush border membrane; certainly it does not result from the low inherent fluidity of the duodenal membrane, because there was no difference between the fluidities of the duodenal and the jejunal membranes under control conditions, whereas their susceptibilities to damage were quite different.

This work was funded by grants from MRC (G84185056CA), University of Newcastle upon Tyne Research Committee (563022), and SmithKline Foundation. We thank Dr J R Greenwell for statistical advice, and Ken Elliott for technical assistance.

\section{References}

1 Israel Y, Salazar I, Rosenmann E. Inhibitory effects of alcohol on intestinal amino acid transport in vivo and in vitro. J Nutr 1968; 96: 499-504.

2 Green RS, MacDermid RG, Scheig RL, Hajjar JJ. Effect of ethanol on amino acid absorption across in vivo rat intestine. Am J Physiol 1981; 241: G176-81.

3 Chang T, Lewis J, Glazko AJ. Effect of ethanol and other alcohols on the transport of amino acids and glucose by everted gut sacs of rat small intestine. Biochim Biophys Acta 1967; 135: 1000-7.

4 Dinda PK, Beck IT, Beck M, McElligott TF. Effect of ethanol on sodium-dependent glucose transport in the small intestine of the hamster. Gastroenterology 1975; 68: $1517-26$.

5 Hunter CK, Treanor LL, Gray JP, Halter SA, Hoyumpa A, Wilson FA. Effects of ethanol in vitro on rat intestinal brush-border membranes. Biochim Biophys Acta 1983; 732: 256-65.

6 Beesley RC. Ethanol inhibits $\mathrm{Na}^{+}$-gradient-dependent uptake of $\mathbf{L}$-amino acids into intestinal brush border membrane vesicles. Dig Dis Sci 1986; 31: 987-92. 
7 Davenport HW. Ethanol damage to canine oxyntic glandular mucosa. Proc Soc Exp Biol Med 1967; 126: $657-62$.

8 Hirst BH. The gastric mucosal barrier. In: Forte JG, ed. Handbook of physiology, The gastrointestinal system III Washington DC: American Physiological Society, in press.

9 Weisbrodt NW, Kienzle M, Cooke AR. Comparative effects of aliphatic alcohols on the gastric mucosa. Proc Soc Exp Biol Med 1973; 142: 450-4.

10 Kessler M, Acuto O, Storelli C, Murer H, Muller M, Semenza G. A modified procedure for the rapid preparation of efficiently transporting vesicles from small intestinal brush border membranes. Their use in investigating some properties of D-glucose and choline transport systems. Biochim Biophys Acta 1978; 506: 136-54.

11 Hirst BH, Forte JG. Redistribution and characterization of $\left(\mathrm{H}^{+}+\mathrm{K}^{+}\right)$-ATPase membranes from resting and stimulated gastric parietal cells. Biochem $J$ 1985; 231: 641-9.

12 Kamino K, Inouye A. Light-scattering studies on brain microsomes. I. Evidence for osmotic behavior. Biochim Biophys Acta 1969; 183: 36-47.

13 Shinitzky M, Barenholz Y. Fluidity parameters of lipid regions determined by fluorescence polarization. Biochim Biophys Acta 1978; 515: 367-94.

14 Wilkes JM, Ballard HJ, Latham JAE, Hirst BH. Gastroduodenal epithelial cells: the role of apical membranes in mucosal protection. In: Reid E, Cook GMW, Luzio JP, eds. Cells, membranes and disease, including renal. New York: Plenum, 1987: 243-54.

15 Bradford MM. A rapid and sensitive method for the quantitation of microgram quantities of protein utilizing the principle of protein-dye binding. Anal Biochem 1976; 72: 248-54.

16 Wilkes JM, Ballard HJ, Dryden DTF, Hirst BH. Proton permeability and lipid dynamics of gastric and duodenal apical membrane vesicles. Am J Physiol 1988. (In press.)

17 Bailey RE, Levine RA, Nandi J, et al. Effects of ethanol on gastric epithelial cell phospholipid dynamics and cellular function. Am J Physiol 1987; 252: G237-43.

18 Bailey RE, Nandi J, Levine RA, Ray TK, Borer PN, Levy GC. NMR studies of pig gastric microsomal $\mathrm{H}^{+}, \mathrm{K}^{+}$-ATPase and phospholipid dynamics: effects of ethanol perturbation. J Biol Chem 1986; 261: 11086-90.

19 Halstead CH, Robles EA, Mezey E. Distribution of ethanol in the human gastrointestinal tract. Am J Clin Nutr 1973; 26: 831-4. 